Published in P. Encarnação, S. Ray-Kaeser, N . Bianquin (Eds.), Guidelines for supporting children with disabilities'play, 2018, pp.67-84 which should be cited to refer to this work.

\title{
7 \\ Which toys and games are appropriate for our children?
}

Sylvie Ray-Kaeser, Odile Perino, Maria Costa, Eleanor Schneider, Vardit Kindler and Andrea Bonarini

\section{Toys and games}

Mainstream toys are 3-Dimensional and sensorial objects that adults give children to support their play activities and which are designed with this intention.

Although toys are the tools of play, play is not an attribute of toys. The play belongs to the player, to the child who wants and needs to play as long as the toy is compatible with his/her functioning and interests.

However, any object can become a toy if the child chooses to play with it and if the safety conditions are guaranteed. For example, a toy can be an object from nature (e.g. pebbles, leaves) or an object from the house (e.g. pan, adult shoe, empty box, paper).

Toys belong to the concrete reality where a child lives. They are essential mediators between a child and his/her environment. They are means for social exchange between children and enable them to play together. Being a mediator between the physical reality and its symbolic representation, toys also empower a child to express his/her feelings, worries or concerns.

In recent years, and thanks to electronics, a more subtle mechanism of interaction has been introduced in some toys. It is possible to add sensors that can perceive 
actions, so that the toy can autonomously react with varying degrees of complexity while maintaining its characteristics. The resulting interaction may enable a different relationship with the toy, not only as an object that can be used at the player's will, or can do exactly what the player wants, but also as an agent that can decide to do what it 'wants'. These toys may provide other play challenges, where the player has to understand how the toy is behaving and adapt his/her activity to that of the toy.

Games are a structured and competitive form of play. Key components of games are rules, which should be understood, freely accepted and followed by the players. Most games involve more than one player, thereby encouraging development of social relationships. Games generally involve mental or physical stimulation, and often both.

In many situations, a game is supported by objects, instrumental to the game, such as cards, a rope, a ball or a computer. The gaming situation can also be abstract as in word games.

Toys and games should be chosen according to the capacities and interests of the play participants in order to enable them to experience playfulness.

There are toys or games for the different types of play:

- Toys for practice play - sensorial toys or toys to manipulate and experiment with the body and encourage experiencing 'cause and effect' (e.g. rattles, toys to squeeze, empty and fill, push toys).

- Toys for symbolic play - toys used to imitate or simulate a situation and play scenario (e.g. a sword, a doll, a kitchen-set).

- Toys for constructive play - toys to assemble, combine and arrange, create (e.g. blocks).

- Games with rules - games to achieve intended objectives by following the rules of the play (e.g. board games, strategy games, video games). 
Ideas of recommended toys by play types

Toys for practice play e.g. 'Pull along Gussy' from Andreu Toys

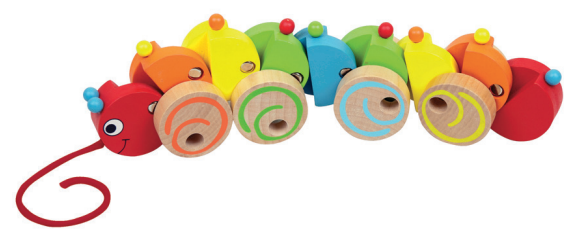

Photo used with permission.

\section{Toys for symbolic play e.g. anatomically correct doll from} Miniland; Parking Garage from PlanToys ${ }^{\circledR}$

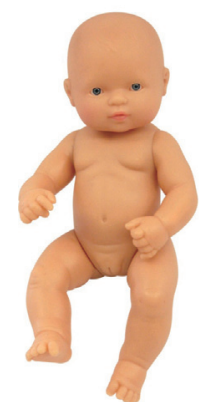

Photo used with permission.

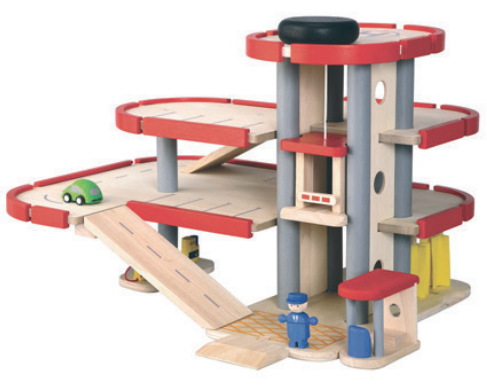

Photo used with permission. 


\section{Toys for constructive play e.g. building bricks from Lego®}

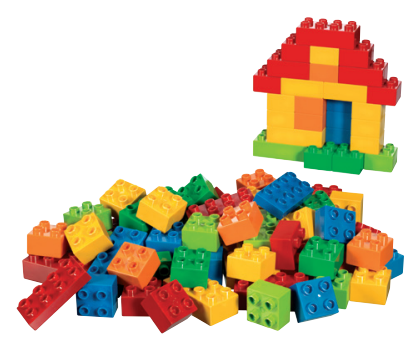

Photo used with permission. (02018 The LEGO Group. ${ }^{2}$

\section{Games with rules e.g. family cooperative game from Haba®}

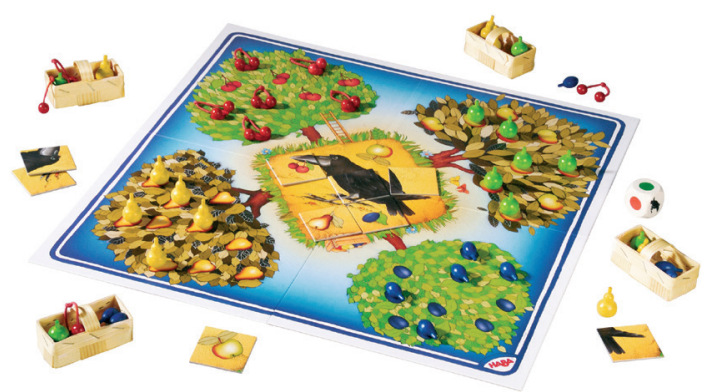

Photo used with permission. OHabermaass $\mathrm{GmbH}$

\footnotetext{
${ }^{2}$ All information is collected and interpreted by its authors and does not represent the opinion of the LEGO Group.
} 


\section{Accessible toys and games for all}

All children differ in their functioning and preferences. Toys and games are accessible when they can be used by all children and when their different components are suitable for all abilities. Inclusive toys and games promote play exchanges between children with different skills and offer the possibility for them to feel a sense of belonging to the community of children players.

Certain elements embedded into the design of toys and games will allow more children to successfully interact and play with them. In order to design a flexible, adjustable, usable toy, multiple options must be present.

Universal Design is a philosophy for designing products that are usable by people with the widest possible range of functional capacities. The more Universal Design features a toy or game has, the more likely it is that it can be used successfully by a broad range of children, including those with disabilities. The general principles of Universal Design can be broadly defined as multiple means of representation, multiple means of use and multiple means of play. Below is a selection of the main principles:

- Toys and games should be accessible, flexible and adjustable.

- Toys and games should offer multiple means of play to be an open source of experiences.

- Toys and games should appeal to children with different abilities, encouraging exploration and discovery.

- Toys and games should offer easy ways to use them successfully.

- The overall structure and pieces of the toy/game should feature usable shapes, dimensions and weights: stable, big, light, allowing it to be grasped in different ways, etc.

- Toys and games should be able to be used in different positions, or be disassembled in modules in order to adjust them to the child. 
- Toys and games should promote interactive actions and stimulate multisensory and multimodal experiences.

- Toys and games should have multiple sensory effects: sounds, lights, pictures, movements, vibrations, scents, textures, colours.

- Toys and games should include adjustable features for sound, height and level of difficulty to adapt to individual users.

- Toys and games should offer written and/or audible messages to adapt to individual user capabilities.

- Toys and games should have a quick and simple means of activation: one that does not require many steps in order to begin play.

- The toy and game components as well as buttons, switches, etc., should be easy to connect, to press, to turn on, fit in, grasp, etc.

- The activation buttons, knobs, connectors, etc., of the toy/game should be highlighted and differentiated from the background.

- The toy and game material should be washable.

In addition to these principles, each toy or game must meet safety standards as well as specific regional legal regulations. Finally, the toys/games should include other specific quality requirements, such as durability, comprehensibility, proper functioning as well as the potential for interaction and popularity, which are all aspects that children value.

Applying Universal Design concepts in the design process guarantees better use of the toy for most children and minimises the need for adaptations. For example, a ball with contrasting colours that vibrates and makes noises when rolled may be appropriate for children with a variety of disabilities including hearing or visual impairments and intellectual disabilities.

When designing a toy with sound signals, one must be sure that there is a volume control or other effects to enable a child with hearing impairments perceive the signals (lights, vibrations, written messages...). 
When designing a toy with accessories or with many pieces, the latter should be sufficiently large, easy to assemble and multi-textured so that a child with visual or motor impairments can play with it.

Reviewing the physical characteristics of a toy can help families and caregivers make a decision about its accessibility for a specific group of children.

- Families can have more success in selecting toys for their children by identifying toys that have been designed with Universal Design concepts in mind. These designs not only address individual barriers, but also offer a combination of features to provide the greatest access to children of all abilities.

\section{For ideas of toys accessible for any child:}

AblePlay ${ }^{\text {TM }}$ toy guide http://www.lekotek.org/images/stories/files/pdf/ ableplay toyguide_FINAL_web.pdf

AlJU toy guide http://www.guiaaiju.com/

EASTIN database on assistive technology http://www.eastin.eu/en/searches/ freetext/summary?freetext=toys

\section{For the assessment of the usability of toys} and games by children with hearing, visual or motor impairments:

The 'TUET - Toys and games Usability Evaluation Tool' developed within the LUDI COST Action. http://www.tuet.eu 


\section{Selecting toys and games}

Well-chosen toys and games, when used in a suitable environment, in a playful context, with play partners, children or adults, have the best chance to allow all children to play for the sake of play.

When selecting a toy or a game for a child, it is important to consider the features of the toy or game, the child's abilities, needs and preferences, the activity promoted by the toy or game and the expected play context. Indeed, even with the best-designed toy or game, it is not guaranteed that a child can and will play. Although toys and games are designed to perform a specific activity, in principle they can be used for play in a variety of ways. When selecting a toy or a game, there is freedom to explore possible alternative ways to use it and reshape the corresponding activity or adapt the toy or game to match the abilities and preferences of the player.

- It is important to remember that the best toy is the one that matches the child's characteristics (skills and abilities, personal preferences) and the play characteristics (level of challenge, demands and requirements of the activity).

Selecting the optimal toy or game requires an analysis of both the child's abilities and preferences as well as an analysis of the toy or game and play itself.

Information can be obtained in two main ways:

a. By asking the child/caregivers questions in order to define a child's needs and preferences in relation to toys/games and level of ability/skill. It is also important to consider the caregivers' preferences and abilities in playing with the child.

b. By observing the child in different play situations looking at a variety of behaviours, using an informal or formal play evaluation when appropriate. 


\section{With regard to elements related to the child}

- What toys does the child prefer and succeed at playing with, and consider fun and enjoyable?

- With whom does the child like to play - alone, with peers, siblings, parents, caregivers?

- What is the preferable play context for the child - indoors or outdoors, the positions for play - moving, sitting or lying?

- Does the child have time for play? Is there a preferred or optimal time during the daily routine? Does the child have time to enjoy and control the play with the toy?

- What toys/materials are available for the child to play with?

- Are there differences in the child's level of performance - persistence, attention, coordination, mobility, communication, wellbeing, enjoyment with different kinds of toys, play partners, play context?

\section{With regard to elements related to the toy/activity}

a. General aspects related to the toy/activity

- For what age group/developmental level is the toy/activity appropriate?

There is an advantage in buying toys that can be used with children of different ages in different ways, for example: balls, building blocks, drawing materials.

- Is the toy typically associated with gender, culture (e.g. dolls, trucks, toys for cooking)?

Lack of gender dependency is preferable to enable more varied use.

- What is the cost of the toy?

If the toy can be used in a variety of ways and is multipurpose, it is likely to be more cost effective. 
- Physical requirements: are any additional materials needed?

- What are the space requirements, large vs small space, level of noise, lighting? Is there room for adjustment?

- How much time is needed for playing with the toy? Can the play activity be broken up into smaller parts and completed over a reasonable period of time? This is a positive aspect.

- How many steps are required to play with the toy?

There is an advantage in toys where there is the potential to modify or grade the steps, varying the level of required mediation in playing.

- Safety precautions: is there potential for physical harm, allergic reaction, or psychological distress?

- What skills are required and what can be facilitated?

- Is there potential for adaptation or modification of the toy to match the child's needs?

b. Specific aspects related to the toy/activity

\section{Motor requirements}

- What is the required position/posture? Can it be changed/modified?

- What mobility, range of movements, dexterity, bilateral hand use and coordination are required? What motor planning is required?

- What strength and endurance are required?

\section{Sensory requirements}

- What sensory input/feedback are required and provided - visual, auditory, touch/pain/temperature, vestibular, proprioceptive, olfactory, taste?

- What sensory discrimination is required? 


\section{Cognitive requirements}

- How much attention span and concentration are needed? Memory? Planning? Problem solving? Level of arousal? Judgment? Understanding? Learning?

\section{Perceptual requirements}

- Does the toy demand identification or matching by size, colour, shape, quantity? Should the player be able to distinguish left from right? Are figure-ground perception, depth perception required?

- How much visual-perceptual-motor integration is required?

\section{Social-emotional and psychological aspects of the toy/activity}

- Is play with the toy structured or unstructured?

- How much creativity and expression are required/allowed/facilitated?

- What motivates the child to engage with the toy?

- How much interaction, cooperation, sharing, turn-taking, conversation are involved? Does the toy/activity involve competition?

- How much choice is allowed?

- Does play with the toy provide a sense of control, competence, achievement?

- How challenging is the toy and how much opportunity is there for success? It is important that the toy/activity provides 'just the right amount of challenge' for the child.

It is important that the child has a balance between high-tech and low-tech toys, as they require different levels of the above-mentioned requirements, such as hand manipulation, hands-on creativity. 


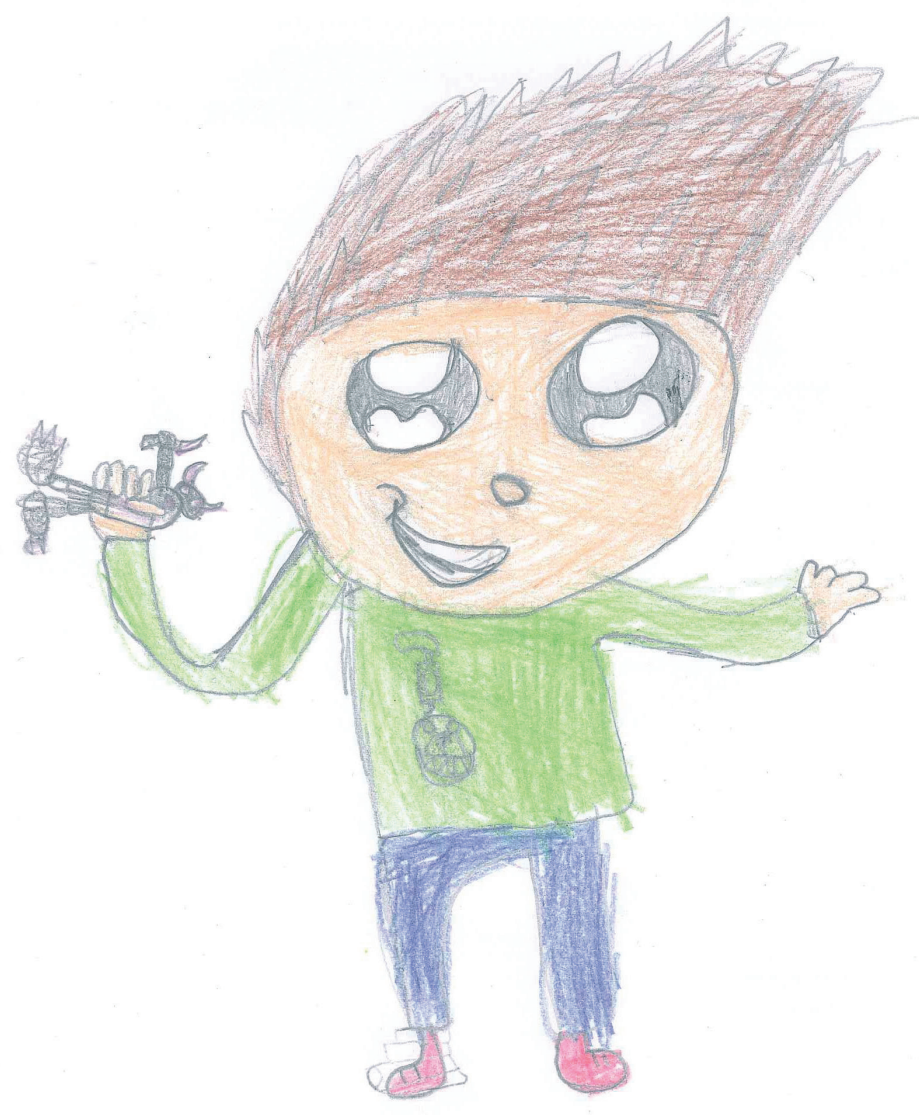

Wences, 8 years old, Spain

\section{Toy or game adaptation}

A child with disabilities might not be able to actively access, explore and take control of the toys and play materials. The toys and play promoted by the toys might not be suitable to his or her abilities.

Universal Design principles applied to toys may not completely eliminate the need 
for specific adaptations of toys. There are some commercially available adapted toys. Usually these are battery-operated toys in which it is possible to connect an external single switch to activate the toy, thus enabling children with motor impairments to use them. However, adapted toys are often expensive. Although they are assistive technology devices and, as such, they are eligible for public funding, many times in practice it is not easy to get funding when funds are scarce and allocation is primarily decided on base of medical considerations.

There are, however, many simple adaptations to assist the child in getting the most out of play. The purpose of these adaptations is usually related to stabilising, extending/building up, highlighting, attaching, confining and/or simplifying the toys/ games.

\section{Stabilising}

Often toys that stay 'in one place' can be easier to use. Materials such as Velcro ${ }^{\circledR}$ fasteners, special grip rubber, or magnetic tape may assist play by securing a toy within the child's reach or vision.

\section{Extending / Building Up}

Materials like playdough lolly sticks or sponge rollers are used to build up certain access features. They help children press buttons or keys that are too small, or make markers and puzzle pieces easier to hold.

\section{Highlighting Materials}

Coloured Velcro® tape, Wikki Stix ${ }^{\circledR}$, coloured masking tape, etc., are materials that can be used to highlight/enhance certain areas on toys, making them easier to locate. They help to simplify toy design and facilitate independent play by children.

\section{Attaching}

Materials such as snaps on fabric tape, elastic straps, or coloured Velcro ${ }^{\circledR}$ straps are used to bring items closer to the child, making reaching, grasping and playing less demanding. 


\section{Confining}

Sometimes items are needed to create play areas that confine several toys. Materials such as hula hoops, box tops or planter bases, can prevent a toy from moving too far away from a child (out of the child's reach or vision). These items create boundaries to help a child control his immediate play environment. Their use may particularly benefit children with visual or physical impairments.

\section{Simplifying}

Try simplifying how play materials are presented to your child. Limiting the number of toy choices and pieces may make play easier. For example, by removing distractions from the play area before play begins. Select 1-2 toys at a time and place them on a contrasting colour surface.

\section{Interfacing}

Technological toys may be difficult to operate and proper interfaces may substitute the original ones: larger operating buttons, properly shaped handles, proper support providing stability, vocal commands instead of gestures or gestures instead of vocal interaction. Since, in some situations, the user can produce liquid secretions and technological tools may suffer from being wet, they could be protected (e.g. a joystick could be inserted in a plastic bag that could guarantee the good operability of the toy).

When a child is unable to play and use the provided toys, an occupational therapist can help you:

- Select toys or games that fit well both the child's interests, needs, abilities, and the family priorities and demands.

- Identify and recommend, for all age groups, accessible and safe toys and games that can challenge the child.

- Adapt or modify the child's toys and games, in order to support his/her participation and pleasure in play. 
- Advise ways of using toys and games while considering the child, home or school routines and habits, and balance of play and work.

- Collaborate with families and educators to create opportunities that encourage the child to play with toys, promoting a sense of wellbeing.

When the required modifications may seem difficult to identify, design and implement, an engineer or technician can help you:

- Modify existing toys to match the player's needs and preferences and make it possible to play with a toy that would otherwise be hard to use.

- Add a dimension to animate the toy so that it can express signals that can be perceived by the player: movements, sounds, and lights. The player can explicitly decide to activate the mechanism. This makes it possible to create a cause-effect relationship that could be used both as a stimulus to induce interest and engagement, and as a reward for well-performed interactive actions.

- Program or re-program the toy to adapt it to the child's abilities, for example, modifications of the toy's actions and changes over time of the toy behaviour to keep the child engaged in the play.

\section{Case study:}

Simple ways for adapting a toy and enabling independent play with an age-appropriate toy - Story of Mark told by his occupational therapist

Mark was 3 years old when I first met him. He was diagnosed with severe cerebral palsy. Mark had a pronounced motor limitation; he was a very verbal and interactive child. He insisted on trying the same activities that his typically developing twin brother played with. He used his right hand (that had very limited movement) for stabilising the toys and his left hand for handling the toys themselves as best he could.

At that time, the favourite toy among Mark's peers was a bubble gun: a plastic toy gun that produced bubbles when the trigger was pressed. This commercial toy was unsuitable to Mark because it needed to be held with two hands: the soap container with one hand and the 'wand' with the other 
hand. It also needed to be stabilised to dip it in the soap solution. It needed fine oral-motor movements to blow air at the wand. Blowing virtual bubbles via a computer program or iPad was not an option for Mark; he insisted on using a 'real' bubble gun.

The solution we found for Mark to enable independent and group play using the bubble gun was adapting the gun for switch use. This meant creating a 'switch adapted toy', i.e. a toy that can be controlled by an external switch thus becoming accessible to all children. This provides a way for the child to make the toy go 'all by itself'. This possibility provides the means to self-generated, active engagement, as the ability to exert control becomes possible. It promotes a proactive attitude of 'I can do it!' leading to increased initiation attempts and empowerment. Using switches with toys develops play skills that include turning them on and off, moving them for social and communicative purposes, and making choices to indicate preferences, not to mention just having fun!

All one needs is a battery switch adaptor, which you can make or buy; you can also reach your Occupational Therapist to help you make one. You then can attach it to any simple battery operated toy of your preference and connect a switch to operate it, as in this example:

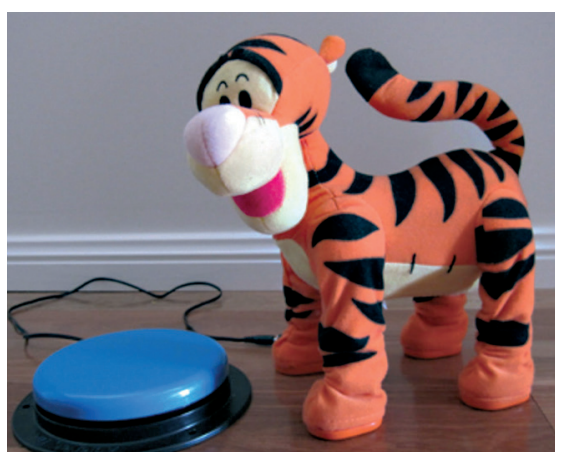

Switch-adapted Tiger

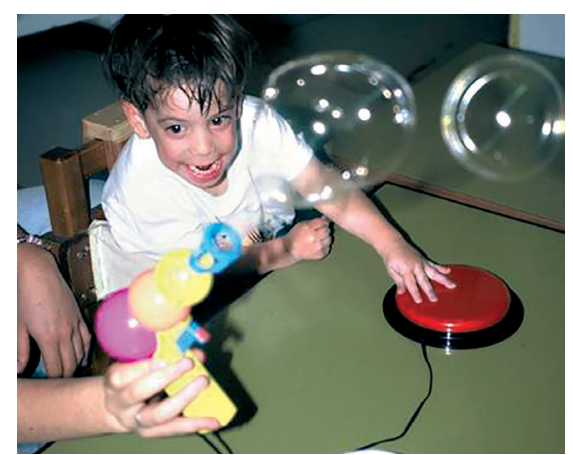

Mark - I can do it by myself !!!

It was Mark's choice to play with the bubble gun and therefore that is the toy we adapted. Mark used his left hand to press the switch that was connected with a battery switch adaptor to the bubble gun, thus enabling him to produce as many bubbles as he wanted and when he wanted! In this particular picture, an adult is holding the toy gun, but it could also have been attached to the table using a variety of kinds of support. 


\section{Playing with toys}

Using mainstream toys with children with disabilities will be easier if they are displayed within a 'play framework'. This framework helps a child play freely, for fun, in confidence and control, and helps parents or caregivers become play mediators.

It comprises several elements:

1 - An area dedicated to play freely that the child knows is his/her special playspace. This area provides a feeling of control and emotional safety. It can be any suitable place where toys can be stored and spread out. Classrooms or rehabilitation rooms are usually unsuitable spaces for free play.

2 - Several separated playspaces, organized according to the toys' categories. For example, one for sensory-motor toys, a second for dolls and symbolic/pretend play, another for construction games and a fourth for rule play games.

3 - Well-chosen challenging toys and games, displayed within playspaces, one by one and not in a jumble, so that the child can easily understand his/her play environment.

4 - Adults as play mediators and belonging to the play framework, where they encourage the child to access and explore the toys by him/herself, and adapt their behaviours so that the child can lead the play. For example, adults may start the play session with the child rediscovering some of the toys that have already been used. Then, gradually introduce different toys that allow the child to recombine mastered skills in new play situations. Please refer to Chapter 5 for more details on the adult's role in play contexts.

5 - Clearly defined rules for use of the playspaces and toys, so that the child can adapt his/her actions and behaviours: for example, toys must not be thrown away. 


\section{Conclusion}

- Toys and games are objects that support children's play.

- There are toys and games for different categories/types of play.

- They should be chosen according to the players' capacities and interests and the social and physical context of play.

- Toys and games with Universal Design features are more likely to be used successfully by children with disabilities.

- When children cannot access and use toys and games, there still are many simple adaptations and possibilities to frame the play in order to assist the child in getting the most out of the play experience.

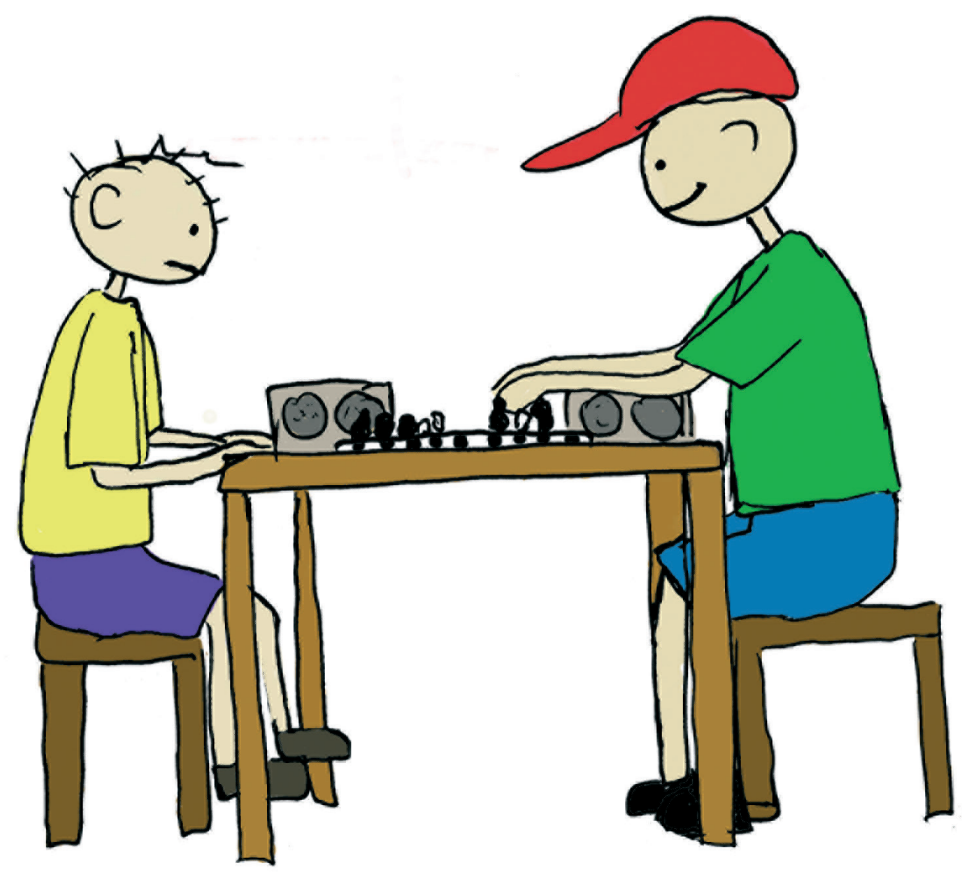

